\title{
A series expansion for generalized harmonic functions
}

\author{
Markus Klintborg ${ }^{1} \cdot$ Anders Olofsson $^{1}$
}

Received: 24 November 2019 / Revised: 27 May 2021 / Accepted: 30 May 2021 / Published online: 11 June 2021 (c) The Author(s) 2021

\section{Abstract}

We consider a class of generalized harmonic functions in the open unit disc in the complex plane. Our main results concern a canonical series expansion for such functions. Of particular interest is a certain individual generalized harmonic function which suitably normalized plays the role of an associated Poisson kernel.

Keywords Harmonic function · Power series · Poisson kernel · Hypergeometric function

Mathematics Subject Classification Primary 31A05; Secondary 33C05 $\cdot 35 \mathrm{~J} 15$

\section{Introduction}

Let $\mathbb{D}$ be the open unit disc in the complex plane $\mathbb{C}$ and denote by $\partial_{z}=\partial / \partial z$ and $\bar{\partial}_{z}=\partial / \partial \bar{z}$ the usual complex partial derivatives. This work is concerned with second order partial differential operators of the form

$$
L_{p, q}=\left(1-|z|^{2}\right) \partial_{z} \bar{\partial}_{z}+p z \partial_{z}+q \bar{z} \bar{\partial}_{z}-p q, \quad z \in \mathbb{D},
$$

where $p, q \in \mathbb{C}$ are complex parameters. Of particular interest are solutions of the associated homogeneous equation

$$
L_{p, q} u=0 \text { in } \mathbb{D} .
$$

We say that a function $u$ is $(p, q)$-harmonic if $u$ is twice continuously differentiable in $\mathbb{D}$ (in symbols $u \in C^{2}(\mathbb{D})$ ) and $L_{p, q} u=0$ in $\mathbb{D}$, where $L_{p, q}$ is as in (0.1). Notice

$\triangle$ Anders Olofsson

anders.olofsson@math.lu.se

Markus Klintborg

kborgmarkus@protonmail.com

1 Mathematics, Faculty of Science, Centre for Mathematical Sciences, Lund University, P.O. Box 118, 22100 Lund, Sweden 
that a function $u$ is $(p, q)$-harmonic if and only if its complex conjugate $\bar{u}$ is $(\bar{q}, \bar{p})$ harmonic. Observe also that a $(0,0)$-harmonic function is a harmonic function in $\mathbb{D}$ in the usual sense.

An interesting example of a $(p, q)$-harmonic function is the function

$$
u_{p, q}(z)=\frac{\left(1-|z|^{2}\right)^{p+q+1}}{(1-z)^{p+1}(1-\bar{z})^{q+1}}, \quad z \in \mathbb{D}
$$

(see Theorem 1.4). Here powers are defined in the usual way using the principal branch of the $\operatorname{logarithm}$, that is, we require that $\log (1)=0$. Notice that the above functions $u_{p, q}$ have the hermitian symmetry property that $\bar{u}_{p, q}=u_{\bar{q}, \bar{p}}$ for $p, q \in \mathbb{C}$.

Recall that elements of the unit circle $\mathbb{T}=\partial \mathbb{D}$ act on the unit disc $\mathbb{D}$ as rotations. On a function level we consider rotation operators

$$
R_{e^{i \theta}} u(z)=u\left(e^{i \theta} z\right), \quad z \in \mathbb{D},
$$

for $e^{i \theta} \in \mathbb{T}$ acting on functions $u$ in $\mathbb{D}$. A basic observation concerning the differential operator $L_{p, q}$ is the commutativity relation

$$
L_{p, q} R_{e^{i \theta}} u=R_{e^{i \theta}} L_{p, q} u, \quad u \in C^{2}(\mathbb{D})
$$

for $e^{i \theta} \in \mathbb{T}$. This latter commutativity relation suggests an analysis of $(p, q)$-harmonic functions using concepts natural to classical Fourier analysis on the unit circle.

Let $\mathbb{Z}$ be the set of integers. For a suitably smooth function $u$ in $\mathbb{D}$ we define its $m$-th homogeneous part by the formula

$$
u_{m}(z)=\frac{1}{2 \pi} \int_{\mathbb{T}} e^{-i m \theta} u\left(e^{i \theta} z\right) d \theta, \quad z \in \mathbb{D},
$$

for $m \in \mathbb{Z}$. Notice that the $m$-th homogeneous part $u_{m}$ of $u$ is the $m$-th Fourier coefficient of the vector-valued function

$$
\mathbb{T} \ni e^{i \theta} \mapsto R_{e^{i \theta}} u
$$

where $R_{e^{i \theta}}$ is as in (0.3).

We set $C^{\infty}(\mathbb{D})=\bigcap_{n=0}^{\infty} C^{n}(\mathbb{D})$, where $C^{n}(\mathbb{D})$ is the space of $n$-times continuously differentiable functions in $\mathbb{D}$ for $n \in \mathbb{N}=\{0,1,2, \ldots\}$. We topologize these spaces in the usual way using the semi-norms

$$
\|u\|_{j, k ; K}=\max _{z \in K}\left|\partial^{j} \bar{\partial}^{k} u(z)\right|,
$$

where $j, k \in \mathbb{N}$ are non-negative integers and $K \subset \mathbb{D}$ is a compact subset of $\mathbb{D}$.

Let us recall the classical hypergeometric function defined by

$$
F(a, b ; c ; z)=\sum_{n=0}^{\infty} \frac{(a)_{n}(b)_{n}}{(c)_{n}} \frac{z^{n}}{n !}, \quad z \in \mathbb{D},
$$


for $a, b, c \in \mathbb{C}$ such that $c \neq-1,-2, \ldots$ Here $(a)_{0}=1$ and

$$
(a)_{n}=a(a+1) \ldots(a+n-1)
$$

for $n=1,2, \ldots$ are Pochhammer symbols.

Let us return to a $(p, q)$-harmonic function $u$. We show that the $m$-th homogeneous part of $u$ has the form

$$
u_{m}(z)=c_{m} F\left(-p, m-q ; m+1 ;|z|^{2}\right) z^{m}, \quad z \in \mathbb{D}
$$

for some $c_{m} \in \mathbb{C}$ when $m \in \mathbb{N}$ is a non-negative integer (see Theorem 4.3). Here $F$ is the hypergeometric function (0.5). Hermitian symmetry leads to a similar formula for $u_{m}$ when $m \in \mathbb{Z}^{-}=\mathbb{Z} \backslash \mathbb{N}$ is a negative integer (see Corollary 4.4). Notice that $u_{m} \in C^{\infty}(\mathbb{D})$ for $m \in \mathbb{Z}$.

A principal result of the present paper concerns the asymptotic behavior of the $m$-th homogeneous part $u_{m}$ of a $(p, q)$-harmonic function $u$. We show that

$$
\underset{|m| \rightarrow \infty}{\lim \sup }\left\|u_{m}\right\|_{j, k ; K}^{1 /|m|}<1
$$

for all $j, k \in \mathbb{N}$ and $K \subset \mathbb{D}$ compact (see Theorem 4.6). This result enables us to use the classical root test to establish absolute convergence of the function series $\sum_{m=-\infty}^{\infty} u_{m}$ in $C^{\infty}(\mathbb{D})$ (see Corollary 4.7).

A further analysis leads to a function series characterization of $(p, q)$-harmonic functions. A function $u$ in $\mathbb{D}$ is $(p, q)$-harmonic if and only if it has the form

$$
\begin{aligned}
u(z)= & \sum_{m=0}^{\infty} c_{m} F\left(-p, m-q ; m+1 ;|z|^{2}\right) z^{m} \\
& +\sum_{m=1}^{\infty} c_{-m} F\left(-q, m-p ; m+1 ;|z|^{2}\right) \bar{z}^{m}, \quad z \in \mathbb{D},
\end{aligned}
$$

for some sequence $\left\{c_{m}\right\}_{m=-\infty}^{\infty}$ of complex numbers such that

$$
\limsup _{|m| \rightarrow \infty}\left|c_{m}\right|^{1 /|m|} \leq 1
$$

(see Theorem 5.1). As mentioned above, the sums in (0.6) are absolutely convergent in the space $C^{\infty}(\mathbb{D})$. As a consequence we have that $u \in C^{\infty}(\mathbb{D})$ if $u$ is $(p, q)$-harmonic (see Corollary 4.9). This characterization of $(p, q)$-harmonic functions improves on a result by Ahern et al. [1, Theorem 2.1] when specialized to our setting.

We then turn to coefficient formulas for $(p, q)$-harmonic functions. We show that

$$
c_{m}=\partial^{m} u(0) / m ! \text { and } c_{-m}=\bar{\partial}^{m} u(0) / m !
$$


for $m \in \mathbb{N}$, where the $c_{m}$ 's are as in (0.6) (see Theorem 5.3). As a consequence, we have that

$$
\begin{aligned}
u(z)= & \sum_{m=0}^{\infty} \frac{\partial^{m} u(0)}{m !} F\left(-p, m-q ; m+1 ;|z|^{2}\right) z^{m} \\
& +\sum_{m=1}^{\infty} \frac{\bar{\partial}^{m} u(0)}{m !} F\left(-q, m-p ; m+1 ;|z|^{2}\right) \bar{z}^{m}, \quad z \in \mathbb{D},
\end{aligned}
$$

whenever $u$ is a $(p, q)$-harmonic function (see Theorem 5.4) as well as a corresponding uniqueness result for such functions (see Corollary 5.5).

Let us return to the function $u_{p, q}$ in (0.2). A calculation of partial derivatives at the origin of the function $u_{p, q}$ leads to the series expansion

$$
\begin{aligned}
u_{p, q}(z)= & \sum_{m=0}^{\infty} \frac{(p+1)_{m}}{m !} F\left(-p, m-q ; m+1 ;|z|^{2}\right) z^{m} \\
& +\sum_{m=1}^{\infty} \frac{(q+1)_{m}}{m !} F\left(-q, m-p ; m+1 ;|z|^{2}\right) \bar{z}^{m}, \quad z \in \mathbb{D}
\end{aligned}
$$

(see Theorem 6.3). This latter series expansion generalizes a well-known partial fraction decomposition formula for the classical Poisson kernel for $\mathbb{D}$ which is obtained for $p=q=0$.

A main contribution of this paper concerns series expansion of $(p, q)$-harmonic functions. Of particular mention is a limit theorem for associated hypergeometric functions:

$$
\lim _{m \rightarrow \infty} F(-p, m-q ; m+1 ; z)=(1-z)^{p}, \quad z \in \mathbb{D}
$$

(see Theorem 2.6). Apart from its intrinsic interest, this limit theorem provides an efficient tool for the study of limit properties of homogeneous parts of $(p, q)$-harmonic functions.

The results of this paper have applications to Poisson integral representations of $(p, q)$-harmonic functions which is possible when $p, q \in \mathbb{C} \backslash \mathbb{Z}^{-}$are such that $\operatorname{Re}(p)+$ $\operatorname{Re}(q)>-1$. In the final section of the paper we comment briefly on the connection to such theory.

We have traced the study of $(p, q)$-harmonic functions back to Daryl Geller [13]. Other significant contributions are those of Ahern and collaborators [1,2]. Our interest in those topics [18-21] arose in connection to standard weights and earlier work of Garabedian [12]. Borichev and Hedenmalm [6] have established a connection to polyharmonic theory. Other recent related papers are [5,8-10,14,16,17,22].

The authors thank the referee for a careful reading of the manuscript. 


\section{The function $u_{p, q}$ is $(p, q)$-harmonic}

Let $u_{p, q}$ be as in (0.2) for some $p, q \in \mathbb{C}$. Observe that the functions $u_{p, q}$ have the hermitian symmetry property $\bar{u}_{p, q}=u_{\bar{q}, \bar{p}}$. Indeed,

$$
\bar{u}_{p, q}(z)=\overline{u_{p, q}(z)}=\frac{\left(1-|z|^{2}\right)^{\bar{p}+\bar{q}+1}}{(1-\bar{z})^{\bar{p}+1}(1-z)^{\bar{q}+1}}=u_{\bar{q}, \bar{p}}(z)
$$

for $z \in \mathbb{D}$. We shall discuss in this section some differentiation formulas for the functions $u_{p, q}$. In particular, we shall show that the function $u_{p, q}$ is $(p, q)$-harmonic (see Theorem 1.4).

Lemma 1.1 Let $u_{p, q}$ be as in (0.2) for some $p, q \in \mathbb{C}$. Then

$$
z \partial u_{p, q}(z)=\left(-(p+q+1) \frac{|z|^{2}}{1-|z|^{2}}+(p+1) \frac{z}{1-z}\right) u_{p, q}(z)
$$

for $z \in \mathbb{D}$.

Proof Differentiating using the product rule for differentiation we have that

$$
\begin{aligned}
\partial u_{p, q}(z)= & \frac{1}{(1-\bar{z})^{q+1}}\left((p+q+1)\left(1-|z|^{2}\right)^{p+q}(-\bar{z}) \frac{1}{(1-z)^{p+1}}\right. \\
& \left.+\left(1-|z|^{2}\right)^{p+q+1}(-(p+1)) \frac{1}{(1-z)^{p+2}}(-1)\right) \\
= & \left(-(p+q+1) \frac{\bar{z}}{1-|z|^{2}}+(p+1) \frac{1}{1-z}\right) u_{p, q}(z)
\end{aligned}
$$

for $z \in \mathbb{D}$, where the last equality is straightforward to check. This yields the conclusion of the lemma.

We next turn to the $\bar{\partial}$-derivative of $u_{p, q}$.

Lemma 1.2 Let $u_{p, q}$ be as in (0.2) for some $p, q \in \mathbb{C}$. Then

$$
\bar{z} \bar{\partial} u_{p, q}(z)=\left(-(p+q+1) \frac{|z|^{2}}{1-|z|^{2}}+(q+1) \frac{\bar{z}}{1-\bar{z}}\right) u_{p, q}(z)
$$

for $z \in \mathbb{D}$.

Proof We shall use the hermitian symmetry property $\bar{u}_{p, q}=u_{\bar{q}, \bar{p}}$. From Lemma 1.1 we have that

$$
z \partial u_{\bar{q}, \bar{p}}(z)=\left(-(\bar{p}+\bar{q}+1) \frac{|z|^{2}}{1-|z|^{2}}+(\bar{q}+1) \frac{z}{1-z}\right) u_{\bar{q}, \bar{p}}(z)
$$

for $z \in \mathbb{D}$. A complex conjugation now yields the conclusion of the lemma. 
Following earlier practice from [20] we denote by $A=z \partial-\bar{z} \bar{\partial}$ the angular derivative. We next calculate the angular derivative of $u_{p, q}$.

Corollary 1.3 Let $u_{p, q}$ be as in (0.2) for some $p, q \in \mathbb{C}$. Then

$$
(z \partial-\bar{z} \bar{\partial}) u_{p, q}(z)=\left((p+1) \frac{z}{1-z}-(q+1) \frac{\bar{z}}{1-\bar{z}}\right) u_{p, q}(z)
$$

for $z \in \mathbb{D}$.

Proof The result is evident from Lemmas 1.1 and 1.2.

We mention that Corollary 1.3 generalizes [20, Theorem 1.11].

Recall the partial fraction formula

$$
\frac{1-|z|^{2}}{|1-z|^{2}}=\frac{z}{1-z}+\frac{\bar{z}}{1-\bar{z}}+1
$$

for the classical Poisson kernel for the unit disc. Formula (1.1) is straightforward to check.

Theorem 1.4 Let $u_{p, q}$ be as in (0.2) for some $p, q \in \mathbb{C}$. Then $L_{p, q} u_{p, q}=0$ in $\mathbb{D}$, where $L_{p, q}$ is as in (0.1).

Proof Recall Lemma 1.2. Notice that the differential operator $z \partial$ satisfies the product rule for differentiation. It is straightforward to check that

$$
z \frac{\partial}{\partial z}\left(\frac{|z|^{2}}{1-|z|^{2}}\right)=\frac{|z|^{2}}{\left(1-|z|^{2}\right)^{2}}
$$

whenever the formula makes sense. Differentiating using the product rule we have that

$$
\begin{aligned}
|z|^{2} \partial \bar{\partial} u_{p, q}(z)= & -(p+q+1) \frac{|z|^{2}}{\left(1-|z|^{2}\right)^{2}} u_{p, q}(z) \\
& +\left(-(p+q+1) \frac{|z|^{2}}{1-|z|^{2}}+(q+1) \frac{\bar{z}}{1-\bar{z}}\right) z \partial u_{p, q}(z)
\end{aligned}
$$

for $z \in \mathbb{D}$. From Lemma 1.1 we now have that

$$
\begin{aligned}
|z|^{2} \partial \bar{\partial} u_{p, q}(z)= & -(p+q+1) \frac{|z|^{2}}{\left(1-|z|^{2}\right)^{2}} u_{p, q}(z) \\
& +\left(-(p+q+1) \frac{|z|^{2}}{1-|z|^{2}}+(q+1) \frac{\bar{z}}{1-\bar{z}}\right) \\
& \times\left(-(p+q+1) \frac{|z|^{2}}{1-|z|^{2}}+(p+1) \frac{z}{1-z}\right) u_{p, q}(z)
\end{aligned}
$$


for $z \in \mathbb{D}$. Expanding the above product we see that

$$
\begin{aligned}
|z|^{2} \partial \bar{\partial} u_{p, q}(z)= & \left(-(p+q+1) \frac{|z|^{2}}{\left(1-|z|^{2}\right)^{2}}+(p+q+1)^{2} \frac{|z|^{4}}{\left(1-|z|^{2}\right)^{2}}\right) u_{p, q}(z) \\
& -(p+q+1) \frac{|z|^{2}}{1-|z|^{2}}\left((p+1) \frac{z}{1-z}+(q+1) \frac{\bar{z}}{1-\bar{z}}\right) u_{p, q}(z) \\
& +(p+1)(q+1) \frac{|z|^{2}}{|1-z|^{2}} u_{p, q}(z)
\end{aligned}
$$

for $z \in \mathbb{D}$. Multiplying by a factor $\left(1-|z|^{2}\right) /|z|^{2}$ we see that

$$
\begin{aligned}
\left(1-|z|^{2}\right) \partial \bar{\partial} u_{p, q}(z)= & \left((p+q+1)^{2} \frac{|z|^{2}}{1-|z|^{2}}-(p+q+1) \frac{1}{1-|z|^{2}}\right) u_{p, q}(z) \\
& -(p+q+1)\left((p+1) \frac{z}{1-z}+(q+1) \frac{\bar{z}}{1-\bar{z}}\right) u_{p, q}(z) \\
& +(p+1)(q+1) \frac{1-|z|^{2}}{|1-z|^{2}} u_{p, q}(z)
\end{aligned}
$$

for $z \in \mathbb{D}$. Notice an appearance of the classical Poisson kernel in the rightmost term above. Using the partial fraction formula (1.1) we have that

$$
\begin{aligned}
\left(1-|z|^{2}\right) \partial \bar{\partial} u_{p, q}(z)= & (p+q+1)\left((p+q) \frac{|z|^{2}}{1-|z|^{2}}-1\right) u_{p, q}(z) \\
& +((p+1)(q+1)-(p+q+1)(p+1)) \frac{z}{1-z} u_{p, q}(z) \\
& +((p+1)(q+1)-(p+q+1)(q+1)) \frac{\bar{z}}{1-\bar{z}} u_{p, q}(z) \\
& +(p+1)(q+1) u_{p, q}(z)
\end{aligned}
$$

for $z \in \mathbb{D}$. A simplification of terms now leads to the formula

$$
\begin{aligned}
\left(1-|z|^{2}\right) \partial \bar{\partial} u_{p, q}(z) & =(p+q+1)(p+q) \frac{|z|^{2}}{1-|z|^{2}} u_{p, q}(z) \\
& -p(p+1) \frac{z}{1-z} u_{p, q}(z)-q(q+1) \frac{\bar{z}}{1-\bar{z}} u_{p, q}(z)+p q u_{p, q}(z)
\end{aligned}
$$

for $z \in \mathbb{D}$. Recall Lemmas 1.1 and 1.2. In view of these two lemmas our latter formula (1.2) says that $L_{p, q} u_{p, q}=0$ in $\mathbb{D}$. 


\section{A sequence of hypergeometric functions}

Let us first consider a second order partial differential operator of the form

$$
L_{p, q ; r}=\left(1-|z|^{2}\right) \partial_{z} \bar{\partial}_{z}+p z \partial_{z}+q \bar{z} \bar{\partial}_{z}-r, \quad z \in \mathbb{D},
$$

where $p, q, r \in \mathbb{C}$ are complex parameters. A principal case is when $r=p q$. Notice that $L_{p, q ; p q}=L_{p, q}$, where $L_{p, q}$ is as in (0.1). The introduction of an additional parameter $r \in \mathbb{C}$ allows for more general operators appearing in the study of conductivity problems, see for instance Calderón [7] or Astala and Päivärinta [4].

We shall evaluate the operator $L_{p, q ; r}$ on a complex-valued function $u$ in the punctured disc $\mathbb{D} \backslash\{0\}$ of the form

$$
u(z)=f\left(|z|^{2}\right) z^{m}, \quad z \in \mathbb{D} \backslash\{0\}
$$

for some $f \in C^{2}(0,1)$ and $m \in \mathbb{Z}$.

We introduce also the ordinary differential operator

$$
H_{a, b ; c}=(1-x) x \frac{d^{2}}{d x^{2}}+(c-[a+b+1] x) \frac{d}{d x}-a b
$$

where $a, b, c \in \mathbb{C}$ are complex parameters. Notice that the famous hypergeometric ordinary differential equation

$$
(1-x) x y^{\prime \prime}(x)+(c-[a+b+1] x) y^{\prime}(x)-a b y(x)=0
$$

takes the form $H_{a, b ; c} y=0$ using the operator $H_{a, b ; c}$.

Theorem 2.1 Let $L_{p, q ; r}$ be as in (2.1) for some $p, q, r \in \mathbb{C}$. Let $u$ be a function of the form (2.2) for some $f \in C^{2}(0,1)$ and $m \in \mathbb{Z}$. Then

$$
L_{p, q ; r} u(z)=z^{m} H_{a, b ; c} f\left(|z|^{2}\right), \quad z \in \mathbb{D} \backslash\{0\},
$$

where $c=m+1$ and

$$
\left\{\begin{array}{c}
a+b=m-p-q, \\
a b=r-p m .
\end{array}\right.
$$

Proof A differentiation shows that

$$
\partial u(z)=m z^{m-1} f\left(|z|^{2}\right)+z^{m} \bar{z} f^{\prime}\left(|z|^{2}\right)
$$

for $z \in \mathbb{D} \backslash\{0\}$, and similarly that

$$
\bar{\partial} u(z)=z^{m+1} f^{\prime}\left(|z|^{2}\right)
$$


for $z \in \mathbb{D} \backslash\{0\}$. Another differentiation gives that

$$
\partial \bar{\partial} u(z)=(m+1) z^{m} f^{\prime}\left(|z|^{2}\right)+z^{m+1} \bar{z} f^{\prime \prime}\left(|z|^{2}\right)
$$

for $z \in \mathbb{D} \backslash\{0\}$. A calculation using these formulas gives that

$$
\begin{aligned}
L_{p, q ; r} u(z)= & \left(1-|z|^{2}\right)\left((m+1) z^{m} f^{\prime}\left(|z|^{2}\right)+z^{m}|z|^{2} f^{\prime \prime}\left(|z|^{2}\right)\right)+q \bar{z} z^{m+1} f^{\prime}\left(|z|^{2}\right) \\
& +p z\left(m z^{m-1} f\left(|z|^{2}\right)+z^{m} \bar{z} f^{\prime}\left(|z|^{2}\right)\right)-r z^{m} f\left(|z|^{2}\right) \\
= & z^{m}\left(|z|^{2}\left(1-|z|^{2}\right) f^{\prime \prime}\left(|z|^{2}\right)+\left(m+1-[m+1-p-q]|z|^{2}\right) f^{\prime}\left(|z|^{2}\right)\right. \\
& \left.-(r-p m) f\left(|z|^{2}\right)\right)=z^{m} H_{a, b ; c} f\left(|z|^{2}\right)
\end{aligned}
$$

for $z \in \mathbb{D} \backslash\{0\}$, where $c=m+1$, the numbers $a$ and $b$ are as in (2.4) and $H_{a, b ; c}$ is as in (2.3). This yields the conclusion of the theorem.

Equations (2.4) say that $a$ and $b$ are the zeros of the quadratic polynomial

$$
P_{p, q ; r ; m}(\lambda)=\lambda^{2}-(m-p-q) \lambda+r-p m, \quad \lambda \in \mathbb{C} .
$$

Notice that

$$
P_{p, q ; r ; m}(\lambda)=(\lambda+p)(\lambda+q-m)+r-p q, \quad \lambda \in \mathbb{C} .
$$

In particular, the zeros of $P_{p, q ; r ; m}$ are $-p$ and $m-q$ in the principal case when $r=p q$.

Theorem 2.1 suggests a natural construction of $(p, q)$-harmonic functions.

Proposition 2.2 Let $p, q \in \mathbb{C}$. Consider the function

$$
u_{m}(z)=F\left(-p, m-q ; m+1 ;|z|^{2}\right) z^{m}, \quad z \in \mathbb{D}
$$

where $m \in \mathbb{N}$ and $F$ is the hypergeometric function (0.5). Then $u_{m}$ is a $(p, q)$-harmonic function.

Proof Clearly $u_{m} \in C^{\infty}(\mathbb{D})$. It is well-known that the hypergeometric function $y=$ $F(a, b ; c ; \cdot)$ satisfies the hypergeometric equation $H_{a, b ; c} y=0$ (see [3, Section 2.3]). The result now follows by Theorem 2.1.

Corollary 2.3 Let $p, q \in \mathbb{C}$. Consider the function

$$
u_{m}(z)=F\left(-q,|m|-p ;|m|+1 ;|z|^{2}\right) \bar{z}^{|m|}, \quad z \in \mathbb{D},
$$

where $m \in \mathbb{Z}^{-}$and $F$ is the hypergeometric function (0.5). Then $u_{m}$ is a $(p, q)$ harmonic function. 
Proof We consider the complex conjugate

$$
\overline{u_{m}(z)}=F\left(-\bar{q},|m|-\bar{p} ;|m|+1 ;|z|^{2}\right) z^{|m|}, \quad z \in \mathbb{D} \text {. }
$$

By Proposition 2.2 we have that $\bar{u}_{m}$ is a $(\bar{q}, \bar{p})$-harmonic function. From hermitian symmetry we conclude that $u_{m}$ is a $(p, q)$-harmonic function.

Following earlier practice, a function $u$ in $\mathbb{D} \backslash\{0\}$ is said to be homogeneous of order $m \in \mathbb{Z}$ with respect to rotations if it has the property that

$$
u\left(e^{i \theta} z\right)=e^{i m \theta} u(z), \quad z \in \mathbb{D} \backslash\{0\}
$$

for $e^{i \theta} \in \mathbb{T}$. Notice that every function $u$ of the form (2.2) is homogeneous of order $m$ with respect to rotations.

Theorem 2.4 Let $p, q \in \mathbb{C}$ and $m \in \mathbb{N}$. Let $u \in C^{2}(\mathbb{D})$ be homogeneous of order $m$ with respect to rotations. Then $u$ is $(p, q)$-harmonic if and only if it has the form

$$
u(z)=c F\left(-p, m-q ; m+1 ;|z|^{2}\right) z^{m}, \quad z \in \mathbb{D},
$$

for some $c \in \mathbb{C}$, where $F$ is the hypergeometric function (0.5).

Proof From Proposition 2.2 we know that every function $u$ of the form $(2.5)$ is $(p, q)$ harmonic.

Assume next that $u$ is $(p, q)$-harmonic. Since $u$ is homogeneous of order $m$, we can put $u$ on the form (2.2) for some $f \in C^{2}(0,1)$. By Theorem 2.1 we have that

$$
H_{-p, m-q, m+1} f(x)=0, \quad 0<x<1,
$$

where $H_{a, b ; c}$ is as in (2.3). Below we shall check that

$$
(m+1) f^{\prime}(x)+p(m-q) f(x)=o\left(1 / x^{m+1}\right)
$$

as $x \rightarrow 0$. Condition (2.6) allows us to apply [18, Proposition 1.3] to conclude that $f$ is a constant multiple of the hypergeometric function $F(-p, m-q ; m+1 ; \cdot)$. This will then complete the proof of the theorem.

We proceed to check (2.6). Recall formula (2.2). Since $u$ is bounded near the origin we have that $f(x)=\mathcal{O}\left(1 / x^{m / 2}\right)$ as $x \rightarrow 0$. A differentiation of (2.2) gives that

$$
\bar{\partial} u(z)=z^{m+1} f^{\prime}\left(|z|^{2}\right), \quad z \in \mathbb{D} \backslash\{0\} .
$$

Since $\bar{\partial} u$ is bounded near the origin we have that $f^{\prime}(x)=\mathcal{O}\left(1 / x^{(m+1) / 2}\right)$ as $x \rightarrow 0$. We have now checked (2.6).

Theorem 2.4 and its proof are modeled on [18, Theorem 2.1]. We have merely supplied some details. 
We shall use the fact that the hypergeometric functions are analytic in $\mathbb{D}$. Let $H(\mathbb{D})$ be the space of analytic functions in $\mathbb{D}$. The space $H(\mathbb{D})$ is topologized in the usual manner using the semi-norms

$$
\|f\|_{K}=\max _{z \in K}|f(z)|
$$

for $K \subset \mathbb{D}$ compact. Convergence in the space $H(\mathbb{D})$ is usually referred to as normal convergence in $\mathbb{D}$.

Recall the terminology that a subset $\mathcal{F}$ of $H(\mathbb{D})$ is called a normal family if every sequence of functions of $\mathcal{F}$ has a subsequence which converges in $H(\mathbb{D})$. The limit function is not required to belong to $\mathcal{F}$. We refer to Conway [11, Chapter VII] for background.

Recall also the binomial series:

$$
F(a, b ; b ; z)=\sum_{n=0}^{\infty} \frac{(a)_{n}}{n !} z^{n}=\frac{1}{(1-z)^{a}}
$$

for $z \in \mathbb{D}$.

Lemma 2.5 Let $p, q \in \mathbb{C}$ and consider the functions

$$
f_{m}(z)=F(-p, m-q ; m+1 ; z), \quad z \in \mathbb{D},
$$

for $m \in \mathbb{N}$, where $F$ is the hypergeometric function (0.5). Then $\mathcal{F}=\left\{f_{m}: m \in \mathbb{N}\right\}$ is a normal family of analytic functions in $\mathbb{D}$.

Proof We shall prove that the functions in $\mathcal{F}$ are uniformly bounded on compact subsets of $\mathbb{D}$. The conclusion of the lemma then follows by a classical result of Montel (see Conway [11, Theorem VII.2.9]).

Let $K \subset \mathbb{D}$ be compact. Since $K \subset \mathbb{D}$ is compact there exists $0<r<1$ such that $\max _{z \in K}|z|<r$. Choose $N$ such that

$$
|1-(q+1) /(m+1)| \leq 1 / r
$$

for $m>N$. Observe that

$$
\frac{(m-q)_{n}}{(m+1)_{n}}=\prod_{k=0}^{n-1} \frac{m-q+k}{m+1+k}=\prod_{k=0}^{n-1}\left(1-\frac{q+1}{m+1+k}\right) .
$$

Therefore $\left|(m-q)_{n} /(m+1)_{n}\right| \leq 1 / r^{n}$ for $n \in \mathbb{N}$ provided $m>N$.

We now estimate the $f_{m}$ 's with $m>N$. From (0.5) we have that

$$
f_{m}(z)=\sum_{n=0}^{\infty} \frac{(-p)_{n}(m-q)_{n}}{(m+1)_{n}} \frac{z^{n}}{n !}, \quad z \in \mathbb{D} .
$$


Notice in this sum an appearance of the quotient $(m-q)_{n} /(m+1)_{n}$ considered in the previous paragraph. From the triangle inequality and the result of the previous paragraph we have that

$$
\left|f_{m}(z)\right| \leq \sum_{n=0}^{\infty} \frac{(|p|)_{n}}{n !} \frac{|z|^{n}}{r^{n}}=\frac{1}{(1-|z| / r)^{|p|}}
$$

for $|z|<r$ and $m>N$, where the last equality follows by the binomial series (2.8). This proves that the functions in $\mathcal{F}$ are uniformly bounded on $K$.

The following limit theorem will be much useful.

Theorem 2.6 Let $p, q \in \mathbb{C}$. Then

$$
\lim _{m \rightarrow \infty} F(-p, m-q ; m+1 ; z)=(1-z)^{p}, \quad z \in \mathbb{D}
$$

with normal convergence, where $F$ is the hypergeometric function (0.5).

Proof Let the $f_{m}$ 's be as in Lemma 2.5. From Lemma 2.5 we have that the set $\mathcal{F}=$ $\left\{f_{m}: m \in \mathbb{N}\right\}$ is a normal family. From (0.5) we have that

$$
f_{m}^{(n)}(0)=\frac{(-p)_{n}(m-q)_{n}}{(m+1)_{n}}
$$

for $m, n \in \mathbb{N}$. Recall that the Pochhammer symbol $(\cdot)_{n}$ is a monic polynomial of degree $n$. Passing to the limit we have that $\lim _{m \rightarrow \infty} f_{m}^{(n)}(0)=(-p)_{n}$ for $n \in \mathbb{N}$.

From (2.8) we have that $f^{(n)}(0)=(-p)_{n}$ for $n \in \mathbb{N}$, where

$$
f(z)=(1-z)^{p}, \quad z \in \mathbb{D} .
$$

A standard argument now yields that $f_{m} \rightarrow f$ in $H(\mathbb{D})$ as $m \rightarrow \infty$. Assume to reach a contradiction that there exists a compact set $K \subset \mathbb{D}$ such that $\left\{f_{m}\right\}_{m=0}^{\infty}$ does not converge uniformly to $f$ on $K$. Passing to a subsequence we can assume that

$$
\max _{z \in K}\left|f_{m_{k}}(z)-f(z)\right| \geq \delta>0
$$

for $k=1,2, \ldots$ Since the set $\mathcal{F}$ is a normal family, we can after passage to another subsequence if necessary, assume that $f_{m_{k}} \rightarrow g$ in $H(\mathbb{D})$ as $k \rightarrow \infty$ for some $g \in H(\mathbb{D})$. From the first paragraph of the proof we have $g^{(n)}(0)=(-p)_{n}$ for $n \in \mathbb{N}$, and a uniqueness argument gives that $g=f$ in $\mathbb{D}$. Thus $f_{m_{k}} \rightarrow f$ in $H(\mathbb{D})$ as $k \rightarrow \infty$, which contradicts (2.9).

We emphasize that Theorem 2.6 appears much natural in view of the binomial series (2.8). 


\section{A generalized power series}

From the product rule for differentiation we have that

$$
(f g)^{(n)}=\sum_{k=0}^{n}\left(\begin{array}{l}
n \\
k
\end{array}\right) f^{(n-k)} g^{(k)}
$$

for, say, $f, g \in C^{n}[0,1)$.

Lemma 3.1 Let $j, k, m \in \mathbb{N}$ be such that $m \geq j$. Let $u$ be a function of the form (2.2) for some $f \in C^{\infty}[0,1)$. Then

$$
\partial^{j} \bar{\partial}^{k} u(z)=z^{m+k-j} \sum_{l=0}^{j}\left(\begin{array}{l}
j \\
l
\end{array}\right) \frac{(m+k) !}{(m+k+l-j) !}|z|^{2 l} f^{(k+l)}\left(|z|^{2}\right)
$$

for $z \in \mathbb{D}$.

Proof Recall (2.2). Differentiating with respect to $\bar{z}$ we have that

$$
\bar{\partial}^{k} u(z)=z^{m+k} f^{(k)}\left(|z|^{2}\right)
$$

for $z \in \mathbb{D}$. Another differentiation using the product rule for differentiation gives that

$$
\begin{aligned}
\partial^{j} \bar{\partial}^{k} u(z) & =\sum_{l=0}^{j}\left(\begin{array}{l}
j \\
l
\end{array}\right) \frac{(m+k) !}{(m+k-(j-l)) !} z^{m+k-(j-l)} f^{(k+l)}\left(|z|^{2}\right) \bar{z}^{l} \\
& =z^{m+k-j} \sum_{l=0}^{j}\left(\begin{array}{l}
j \\
l
\end{array}\right) \frac{(m+k) !}{(m+k+l-j) !}|z|^{2 l} f^{(k+l)}\left(|z|^{2}\right)
\end{aligned}
$$

for $z \in \mathbb{D}$. This completes the proof of the lemma.

Let $C^{\infty}(\mathbb{D})$ be the set of smooth complex-valued functions in the unit disc $\mathbb{D}$. The space $C^{\infty}(\mathbb{D})$ is topologized by means of the family of semi-norms

$$
\|u\|_{j, k ; K}=\max _{z \in K}\left|\partial^{j} \bar{\partial}^{k} u(z)\right|
$$

where $j, k \in \mathbb{N}$ and $K \subset \mathbb{D}$ is compact. Recall that $u_{m} \rightarrow u$ in $C^{\infty}(\mathbb{D})$ as $m \rightarrow \infty$ means that $\lim _{m \rightarrow \infty}\left\|u_{m}-u\right\|_{j, k ; K}=0$ for all $j, k \in \mathbb{N}$ and $K \subset \mathbb{D}$ compact.

A (formal) series $\sum_{m=0}^{\infty} u_{m}$ of functions $u_{m} \in C^{\infty}(\mathbb{D})$ for $m \in \mathbb{N}$ is said to be absolutely convergent in $C^{\infty}(\mathbb{D})$ if

$$
\sum_{m=0}^{\infty}\left\|u_{m}\right\|_{j, k ; K}<+\infty
$$


whenever $j, k \in \mathbb{N}$ and $K \subset \mathbb{D}$ is compact. By completeness of $C^{\infty}(\mathbb{D})$ we have that every series absolutely convergent in $C^{\infty}(\mathbb{D})$ is convergent in $C^{\infty}(\mathbb{D})$.

Theorem 3.2 Let $\left\{f_{m}\right\}_{m=0}^{\infty}$ be a sequence in $C^{\infty}[0,1)$ such that

$$
\limsup _{m \rightarrow \infty}\left(\max _{0 \leq x \leq r}\left|f_{m}^{(n)}(x)\right|\right)^{1 / m} \leq 1
$$

for $n \in \mathbb{N}$ and $0<r<1$. Set

$$
u_{m}(z)=f_{m}\left(|z|^{2}\right) z^{m}, \quad z \in \mathbb{D}
$$

for $m=0,1,2, \ldots$ Then $\lim \sup _{m \rightarrow \infty}\left\|u_{m}\right\|_{j, k ; K}^{1 / m}<1$ for all $j, k \in \mathbb{N}$ and $K \subset \mathbb{D}$ compact, where $\|\cdot\|_{j, k ; K}$ is as in (3.1).

Proof Fix $j, k \in \mathbb{N}$ and $K \subset \mathbb{D}$ compact. Set $r=\max _{z \in K}|z|<1$. From Lemma 3.1 we have that

$$
\partial^{j} \bar{\partial}^{k} u_{m}(z)=z^{m+k-j} \sum_{l=0}^{j}\left(\begin{array}{l}
j \\
l
\end{array}\right) \frac{(m+k) !}{(m+k+l-j) !}|z|^{2 l} f_{m}^{(k+l)}\left(|z|^{2}\right)
$$

for $z \in \mathbb{D}$ provided $m \geq j$. We next apply the triangle inequality to see that

$$
\begin{aligned}
\left\|u_{m}\right\|_{j, k ; K} & \leq r^{m+k-j}\left(\sum_{l=0}^{j}\left(\begin{array}{l}
j \\
l
\end{array}\right) \frac{(m+k) !}{(m+k+l-j) !} r^{2 l}\right) \max _{k \leq n \leq j+k}\left\|f_{m}^{(n)}\right\|_{\left[0, r^{2}\right]} \\
& \leq r^{m+k-j}(m+k)^{j}\left(1+r^{2}\right)^{j} \max _{k \leq n \leq j+k}\left\|f_{m}^{(n)}\right\|_{\left[0, r^{2}\right]}
\end{aligned}
$$

for $m \geq j$, where we have used the notation (2.7). We shall next apply the $m$-th root to (3.2) and pass to the limit as $m \rightarrow \infty$. In view of the assumption on the sequence $\left\{f_{m}\right\}_{m=0}^{\infty}$ we conclude from (3.2) that

$$
\limsup _{m \rightarrow \infty}\left\|u_{m}\right\|_{j, k ; K}^{1 / m} \leq r
$$

Since $0<r<1$, this yields the conclusion of the theorem.

From the conclusion of Theorem 3.2 we have that $\sum_{m=0}^{\infty}\left\|u_{m}\right\|_{j, k ; K}<+\infty$ whenever $j, k \in \mathbb{N}$ and $K \subset \mathbb{D}$ is compact. Thus the series $\sum_{m=0}^{\infty} u_{m}$ is absolutely convergent in $C^{\infty}(\mathbb{D})$.

We can think of a function series

$$
\sum_{m=0}^{\infty} f_{m}\left(|z|^{2}\right) z^{m}
$$


as a vector-valued power series. The sequence of coefficients $\left\{f_{m}\right\}_{m=0}^{\infty}$ in (3.3) is now a sequence of functions in $C^{\infty}[0,1)$. From this point of view, Theorem 3.2 generalizes a well-known root criteria for power series (see for instance Conway [11, Theorem III.1.3]).

Of particular concern to us are sequences $\left\{f_{m}\right\}_{m=0}^{\infty}$ of the form

$$
f_{m}(x)=F(-p, m-q ; m+1 ; x), \quad 0 \leq x<1,
$$

for $m \in \mathbb{N}$, where $p, q \in \mathbb{C}$ and $F$ is the hypergeometric function (0.5). We next observe that Theorem 2.6 guarantees that every such sequence $\left\{f_{m}\right\}_{k=0}^{\infty}$ satisfies the assumption of Theorem 3.2.

Proposition 3.3 Let $p, q \in \mathbb{C}$. Then

$$
\limsup _{m \rightarrow \infty}\left(\max _{0 \leq x \leq r}\left|F^{(n)}(-p, m-q ; m+1 ; x)\right|\right)^{1 / m} \leq 1
$$

for $n \in \mathbb{N}$ and $0<r<1$, where $F$ is the hypergeometric function (0.5).

Proof Recall the fact that the complex derivative $f \mapsto f^{\prime}$ is continuous in the topology of normal convergence of analytic functions (see Conway [11, Theorem VII.2.1]). Recall Montel's theorem characterizing normal families of analytic functions (see Conway [11, Theorem VII.2.9]). In view of these two results, the proposition follows from Theorem 2.6.

\section{Analysis of homogeneous parts}

Notice that the rotation operators $\mathbb{T} \ni e^{i \theta} \mapsto R_{e^{i \theta}}$ from (0.3) have the group properties that $R_{1}=I$ is the identity and

$$
R_{e^{i(\theta+\tau)}}=R_{e^{i \theta}} R_{e^{i \tau}}
$$

for $e^{i \theta}, e^{i \tau} \in \mathbb{T}$.

Recall the notion of homogeneity with respect to rotations made precise in the paragraph before Theorem 2.4. Observe that a function $u$ in $\mathbb{D}$ is homogeneous of order $m \in \mathbb{Z}$ with respect to rotations if and only if $R_{e^{i \theta}} u=e^{i m \theta} u$ for $e^{i \theta} \in \mathbb{T}$.

Proposition 4.1 Let $u_{m}$ be the $m$-th homogeneous part of $u \in C^{n}(\mathbb{D})$ for some $m \in \mathbb{Z}$, where $n \in \mathbb{N} \cup\{\infty\}$. Then $u_{m} \in C^{n}(\mathbb{D})$ and $u_{m}$ is homogeneous of order $m$ with respect to rotations.

Proof Differentiations under the integral in (0.4) show that $u_{m} \in C^{n}(\mathbb{D})$. From formula (0.4) we have that

$$
u_{m}=\frac{1}{2 \pi} \int_{\mathbb{T}} e^{-i m \tau} R_{e^{i \tau}} u d \tau
$$


in a vector-valued sense. Let $e^{i \theta} \in \mathbb{T}$. From the group property of rotation operators we have that

$$
R_{e^{i \theta}} u_{m}=\frac{1}{2 \pi} \int_{\mathbb{T}} e^{-i m \tau} R_{e^{i \theta}} R_{e^{i \tau}} u d \tau=\frac{1}{2 \pi} \int_{\mathbb{T}} e^{-i m \tau} R_{e^{i(\theta+\tau)}} u d \tau
$$

in a vector-valued sense. A change of variables now gives that

$$
R_{e^{i \theta} u_{m}}=\frac{e^{i m \theta}}{2 \pi} \int_{\mathbb{T}} e^{-i m \tau} R_{e^{i \tau}} u d \tau=e^{i m \theta} u_{m} .
$$

Since $e^{i \theta} \in \mathbb{T}$ is arbitrary, this yields that $u_{m}$ is homogeneous of order $m$ with respect to rotations.

Below we shall make use of the commutativity relation

$$
L_{p, q} R_{e^{i \theta}} u=R_{e^{i \theta}} L_{p, q} u, \quad u \in C^{2}(\mathbb{D})
$$

for $e^{i \theta} \in \mathbb{T}$. In order to prove (4.2) it suffices to check that the differential operators $z \partial$ and $\bar{z} \bar{\partial}$ commute with the rotations $R_{e^{i \theta}}$ which is evident.

We now return to $(p, q)$-harmonic functions.

Proposition 4.2 Let $p, q \in \mathbb{C}$. Let $u$ be $a(p, q)$-harmonic function and denote by $u_{m}$ its $m$-th homogeneous part for some $m \in \mathbb{Z}$. Then $u_{m}$ is $(p, q)$-harmonic.

Proof From Proposition 4.1 we have that $u_{m} \in C^{2}(\mathbb{D})$ since $u \in C^{2}(\mathbb{D})$. Recall formula (4.1). Applying the operator $L_{p, q}$ we have that

$$
L_{p, q} u_{m}=\frac{1}{2 \pi} \int_{\mathbb{T}} e^{-i m \tau} L_{p, q} R_{e^{i \tau}} u d \tau
$$

in a vector-valued sense. We now use the commutativity relation (4.2) to conclude that

$$
L_{p, q} u_{m}=\frac{1}{2 \pi} \int_{\mathbb{T}} e^{-i m \tau} R_{e^{i \tau}} L_{p, q} u d \tau=0,
$$

where the last equality is evident since $u$ is $(p, q)$-harmonic.

Let us next calculate the homogeneous parts of a $(p, q)$-harmonic function.

Theorem 4.3 Let $p, q \in \mathbb{C}$. Let $u$ be $(p, q)$-harmonic function and denote by $u_{m}$ its $m$-th homogeneous part for some $m \in \mathbb{N}$. Then

$$
u_{m}(z)=c_{m} F\left(-p, m-q ; m+1 ;|z|^{2}\right) z^{m}, \quad z \in \mathbb{D}
$$

for some $c_{m} \in \mathbb{C}$, where $F$ is the hypergeometric function (0.5). 
Proof By Proposition 4.1, the function $u_{m}$ is homogeneous of order $m$ with respect to rotations. By Proposition 4.2, the function $u_{m}$ is $(p, q)$-harmonic. The result now follows from Theorem 2.4.

Corollary 4.4 Let $p, q \in \mathbb{C}$. Let $u$ be $(p, q)$-harmonic function and denote by $u_{m}$ its $m$-th homogeneous part for some $m \in \mathbb{Z} \backslash \mathbb{Z}^{+}$. Then

$$
u_{m}(z)=c_{m} F\left(-q,|m|-p ;|m|+1 ;|z|^{2}\right) \bar{z}^{|m|}, \quad z \in \mathbb{D},
$$

for some $c_{m} \in \mathbb{C}$, where $F$ is the hypergeometric function (0.5).

Proof The complex conjugate $\bar{u}_{m}$ is the $-m=|m|$-th homogeneous part of the function $\bar{u}$. By hermitian symmetry, the function $\bar{u}$ is $(\bar{q}, \bar{p})$-harmonic. From Theorem 4.3 we have that

$$
\overline{u_{m}(z)}=a_{m} F\left(-\bar{q},|m|-\bar{p} ;|m|+1 ;|z|^{2}\right) z^{|m|}, \quad z \in \mathbb{D},
$$

for some $a_{m} \in \mathbb{C}$. A complex conjugation now yields (4.4) with $c_{m}=\bar{a}_{m}$.

As a by-product from Theorem 4.3 and Corollary 4.4 we have that $u_{m} \in C^{\infty}(\mathbb{D})$ for $m \in \mathbb{Z}$ if $u$ is $(p, q)$-harmonic.

Recall formula (0.4) for the homogeneous parts of a function $u$ in $\mathbb{D}$. From the triangle inequality we have that

$$
\sup _{|z|=r}\left|u_{m}(z)\right| \leq \sup _{|z|=r}|u(z)|
$$

for $0<r<1$ and $m \in \mathbb{Z}$. Notice that (4.5) generalizes a well-known bound for Fourier coefficients.

We next estimate the constants $c_{m}$ appearing in Theorem 4.3 or Corollary 4.4.

Lemma 4.5 Let $p, q \in \mathbb{C}$. Let $u$ be a $(p, q)$-harmonic function and consider its $m$-th homogeneous part $u_{m}$ for $m \in \mathbb{Z}$. Let $c_{m}$ be as in (4.4) or (4.4) depending on whether $m \in \mathbb{N}$ or $m \in \mathbb{Z}^{-}$. Then $\lim \sup _{|m| \rightarrow \infty}\left|c_{m}\right|^{1 /|m|} \leq 1$.

Proof For simplicity we consider the case $m \in \mathbb{N}$. The case $m \in \mathbb{Z}^{-}$is analogous or follows by hermitian symmetry. Let $0<r<1$. From (4.4) and (4.5) we have that

$$
\left|c_{m}\right|\left|F\left(-p, m-q ; m+1 ; r^{2}\right)\right| r^{m} \leq \max _{|z|=r}|u(z)|
$$

for $m \in \mathbb{N}$. From Theorem 2.6 we have that

$$
\lim _{m \rightarrow \infty} F\left(-p, m-q ; m+1 ; r^{2}\right)=\left(1-r^{2}\right)^{p} \neq 0 .
$$

Therefore, a passage to the limit in (4.6) gives that

$$
\limsup _{m \rightarrow \infty}\left|c_{m}\right|^{1 / m} \leq 1 / r
$$


Since $0<r<1$ is arbitrary we conclude that $\limsup _{m \rightarrow \infty}\left|c_{m}\right|^{1 / m} \leq 1$. This yields the conclusion of the lemma.

The following theorem is our main result about the asymptotic behavior of the homogeneous parts of a $(p, q)$-harmonic function.

Theorem 4.6 Let $p, q \in \mathbb{C}$. Let $u$ be a $(p, q)$-harmonic function and denote by $u_{m}$ its $m$-th homogeneous part for $m \in \mathbb{Z}$. Then $u_{m} \in C^{\infty}(\mathbb{D})$ for $m \in \mathbb{Z}$ and

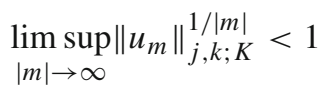

for all $j, k \in \mathbb{N}$ and $K \subset \mathbb{D}$ compact.

Proof From Theorem 4.3 and Corollary 4.4 we have that $u_{m} \in C^{\infty}(\mathbb{D})$ for $m \in \mathbb{Z}$.

We consider first the case $m \in \mathbb{N}$. From Theorem 4.3 we have (4.4) for $m \in \mathbb{N}$, where $c_{m} \in \mathbb{C}$. Notice that the function $u_{m}$ in (4.4) is constructed from the function

$$
f_{m}(x)=c_{m} F(-p, m-q ; m+1 ; x), \quad 0 \leq x<1,
$$

as in Theorem 3.2. From Proposition 3.3 and Lemma 4.5 we have that the assumption of Theorem 3.2 is satisfied. An application of Theorem 3.2 yields the conclusion that $\lim \sup _{m \rightarrow \infty}\left\|u_{m}\right\|_{j, k ; K}^{1 / m}<1$ for $j, k \in \mathbb{N}$ and $K \subset \mathbb{D}$ compact.

We next consider the case $m \in \mathbb{Z}^{-}$. The complex conjugate $\bar{u}_{m}$ of $u_{m}$ is the $(-m)$-th homogeneous part of the $(\bar{q}, \bar{p})$-harmonic function $\bar{u}$. From the result of the previous paragraph we thus have that

$$
\limsup _{m \rightarrow-\infty}\left\|u_{m}\right\|_{j, k ; K}^{1 /|m|}=\limsup _{m \rightarrow \infty}\left\|(\bar{u})_{m}\right\|_{k, j ; K}^{1 / m}<1
$$

for $j, k \in \mathbb{N}$ and $K \subset \mathbb{D}$ compact. This completes the proof of the theorem.

We point out that Theorem 2.6 forms an integral part in the proof of Theorem 4.6. Recall the notion of absolute convergence in $C^{\infty}(\mathbb{D})$, see the paragraph just before Theorem 3.2.

Corollary 4.7 Let $p, q \in \mathbb{C}$. Let $u$ be $a(p, q)$-harmonic function with $m$-th homogeneous part $u_{m}$ for $m \in \mathbb{Z}$. Then the function series $\sum_{m=-\infty}^{\infty} u_{m}$ is absolutely convergent in $C^{\infty}(\mathbb{D})$.

Proof Theorem 4.6 allows us to apply the root test to conclude that

$$
\sum_{m=-\infty}^{\infty}\left\|u_{m}\right\|_{j, k ; K}<+\infty
$$

for $j, k \in \mathbb{N}$ and $K \subset \mathbb{D}$ compact.

The following lemma is well-known but included for the sake of completeness. 
Lemma 4.8 Let $u \in C^{n}(\mathbb{D})$ for some $n \in \mathbb{N}$ and denote by $u_{m}$ its $m$-th homogeneous part for $m \in \mathbb{Z}$. Then

$$
u=\lim _{N \rightarrow+\infty} \sum_{m=-N}^{N}\left(1-\frac{|m|}{N+1}\right) u_{m}
$$

in $C^{n}(\mathbb{D})$.

Proof It is straightforward to check that

$$
\sum_{m=-N}^{N}\left(1-\frac{|m|}{N+1}\right) u_{m}=\frac{1}{2 \pi} \int_{\mathbb{T}} K_{N}\left(e^{i \theta}\right) R_{e^{i \theta}} u d \theta
$$

in a vector-valued sense, where

$$
K_{N}\left(e^{i \theta}\right)=\sum_{m=-N}^{N}\left(1-\frac{|m|}{N+1}\right) e^{i m \theta}, \quad e^{i \theta} \in \mathbb{T},
$$

is the Fejér kernel. It is well-known that the $K_{N}$ 's are non-negative and form an approximate identity as $N \rightarrow \infty$. Since $u \in C^{n}(\mathbb{D})$, it is well-known that the function

$$
\mathbb{T} \ni e^{i \theta} \mapsto R_{e^{i \theta}} u \in C^{n}(\mathbb{D})
$$

is continuous from $\mathbb{T}$ into $C^{n}(\mathbb{D})$. The proof is now completed by a standard argument. We refer to Katznelson [15, Section I.2] for details.

We think of Lemma 4.8 as a version of Fejér's theorem adapted to our context.

We next deduce that $(p, q)$-harmonic functions belong to the space $C^{\infty}(\mathbb{D})$.

Corollary 4.9 Let $p, q \in \mathbb{C}$. Let $u$ be $a(p, q)$-harmonic function. Then $u \in C^{\infty}(\mathbb{D})$ and $u=\sum_{m=-\infty}^{\infty} u_{m}$ in $C^{\infty}(\mathbb{D})$, where $u_{m}$ is the $m$-th homogeneous part of $u$ for $m \in \mathbb{Z}$.

Proof From Lemma 4.8 we have that

$$
u=\lim _{N \rightarrow+\infty} \sum_{m=-N}^{N}\left(1-\frac{|m|}{N+1}\right) u_{m}
$$

in $C^{2}(\mathbb{D})$ since $u$ has such regularity. From Corollary 4.7 we know that the function series $\sum_{m=-\infty}^{\infty} u_{m}$ is absolutely convergent in $C^{\infty}(\mathbb{D})$. We can thus omit the convergence factors in (4.7) and deduce that $u=\sum_{m=-\infty}^{\infty} u_{m}$ in $C^{\infty}(\mathbb{D})$. By completeness we have that $u \in C^{\infty}(\mathbb{D})$. 


\section{A series expansion of harmonic functions}

The analysis from Sect. 4 leads to a natural function series description of $(p, q)$ harmonic functions.

Theorem 5.1 Let $p, q \in \mathbb{C}$. Then $u$ is $a(p, q)$-harmonic function if and only if it has the form

$$
\begin{aligned}
u(z)= & \sum_{m=0}^{\infty} c_{m} F\left(-p, m-q ; m+1 ;|z|^{2}\right) z^{m} \\
& +\sum_{m=1}^{\infty} c_{-m} F\left(-q, m-p ; m+1 ;|z|^{2}\right) \bar{z}^{m}, \quad z \in \mathbb{D}
\end{aligned}
$$

for some sequence $\left\{c_{m}\right\}_{m=-\infty}^{\infty}$ of complex numbers such that

$$
\limsup _{|m| \rightarrow \infty}\left|c_{m}\right|^{1 /|m|} \leq 1
$$

where $F$ is the hypergeometric function (0.5). Moreover, the sums in (5.1) are absolutely convergent in the space $C^{\infty}(\mathbb{D})$ when (5.2) holds.

Proof Consider first a (formal) function series of the form (5.1) with coefficient sequence $\left\{c_{m}\right\}_{m=-\infty}^{\infty}$ satisfying (5.2). From Theorem 3.2 and Proposition 3.3 we have that

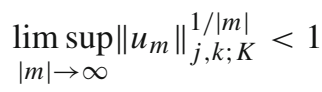

for $j, k \in \mathbb{N}$ and $K \subset \mathbb{D}$ compact, where

$$
u_{m}(z)=c_{m} F\left(-p, m-q ; m+1 ;|z|^{2}\right) z^{m}, \quad z \in \mathbb{D}
$$

for $m \in \mathbb{N}$ and

$$
u_{m}(z)=c_{m} F\left(-q,|m|-p ;|m|+1 ;|z|^{2}\right) \bar{z}^{|m|}, \quad z \in \mathbb{D},
$$

for $m \in \mathbb{Z}^{-}$. The root test now applies to show that the series $\sum_{m=-\infty}^{\infty} u_{m}$ in (5.1) is absolutely convergent in $C^{\infty}(\mathbb{D})$. This defines a function $u=\sum_{m=-\infty}^{\infty} u_{m}$ by (5.1). From Proposition 2.2 and Corollary 2.3 we have that each term $u_{m}$ in $(5.1)$ is $(p, q)$ harmonic. Since the series expansion (5.1) converges in the space $C^{\infty}(\mathbb{D})$, we have that $u \in C^{\infty}(\mathbb{D})$. Applying the operator $L_{p, q}$ we have $L_{p, q} u=\sum_{m=-\infty}^{\infty} L_{p, q} u_{m}=0$ in $C^{\infty}(\mathbb{D})$, which shows that $u$ is $(p, q)$-harmonic.

Consider next a $(p, q)$-harmonic function $u$. We proceed to derive (5.1). Denote by $u_{m}$ the $m$-th homogeneous part of $u$ for $m \in \mathbb{Z}$. From Theorem 4.3 we have (4.4) for $m \in \mathbb{N}$ and from Corollary 4.4 we have (4.4) for $m \in \mathbb{Z}^{-}$, where $c_{m} \in \mathbb{C}$ for $m \in \mathbb{Z}$. From Corollary 4.9 we have (5.1) in the form that $u=\sum_{m=-\infty}^{\infty} u_{m}$ in $C^{\infty}(\mathbb{D})$. 
By Corollary 4.7 we have that the function series $\sum_{m=-\infty}^{\infty} u_{m}$ in (5.1) is absolutely convergent in $C^{\infty}(\mathbb{D})$. From Lemma 4.5 we have that (5.2) holds.

We emphasize that the function series (5.1) has an interpretation of homogeneous expansion of the $(p, q)$-harmonic function $u$.

We mention that Theorem 5.1 improves on a result by Ahern et al. [1, Theorem 2.1] when specialized to the present setting. Theorem 5.1 contains also some earlier results of Olofsson and Wittsten. Theorem 5.1 with $(p, q)=(0, \alpha), \alpha \in \mathbb{R}$, yields [21, Theorem 1.2]. Theorem 5.1 with $(p, q)=(\alpha / 2, \alpha / 2), \alpha \in \mathbb{R}$, yields [18, Theorem 2.2].

We shall next turn to formulas for the coefficients $c_{m}$ appearing in (5.1).

Proposition 5.2 Let $p, q \in \mathbb{C}$. Let $u$ be a $(p, q)$-harmonic function of the form (5.1) for some sequence $\left\{c_{m}\right\}_{m=-\infty}^{\infty}$ of complex numbers satisfying (5.2). Then

$$
c_{m}=\lim _{r \rightarrow 0} \frac{1}{2 \pi r^{|m|}} \int_{\mathbb{T}} u\left(r e^{i \theta}\right) e^{-i m \theta} d \theta
$$

for $m \in \mathbb{Z}$.

Proof We consider the case $m \in \mathbb{N}$. Using the expansion (5.1) it is straightforward to check that

$$
\frac{1}{2 \pi} \int_{\mathbb{T}} u\left(r e^{i \theta}\right) e^{-i m \theta} d \theta=c_{m} F\left(-p, m-q ; m+1 ; r^{2}\right) r^{m}
$$

for $0<r<1$. We next divide by $r^{m}$ and pass to the limit to see that

$$
\lim _{r \rightarrow 0} \frac{1}{2 \pi r^{m}} \int_{\mathbb{T}} u\left(r e^{i \theta}\right) e^{-i m \theta} d \theta=c_{m} F(-p, m-q ; m+1 ; 0)=c_{m} .
$$

This yields the conclusion of the proposition when $m \in \mathbb{N}$.

The remaining case $m \in \mathbb{Z} \backslash \mathbb{N}$ is proved similarly or follows by hermitian symmetry. We omit the details.

The integral quantity

$$
I_{m}(r)=\frac{1}{2 \pi r^{|m|}} \int_{\mathbb{T}} u\left(r e^{i \theta}\right) e^{-i m \theta} d \theta
$$

in Proposition 5.2 is naturally thought of as a line integral. In fact,

$$
I_{m}(r)=\frac{1}{2 \pi i} \oint_{|z|=r} \frac{u(z)}{z^{m+1}} d z \text { and } I_{-m}(r)=-\frac{1}{2 \pi i} \oint_{|z|=r} \frac{u(z)}{\bar{z}^{m+1}} d \bar{z}
$$

for $m \in \mathbb{N}$ and $0<r<1$, where the circle of integration is traversed once in positive direction. These formulas are straightforward to check. 
Theorem 5.3 Let $p, q \in \mathbb{C}$. Let $u$ be $a(p, q)$-harmonic function of the form (5.1) for some sequence $\left\{c_{m}\right\}_{m=-\infty}^{\infty}$ of complex numbers satisfying (5.2). Then

$$
c_{m}=\partial^{m} u(0) / m ! \text { and } c_{-m}=\bar{\partial}^{m} u(0) / m !
$$

for $m \in \mathbb{N}$.

Proof We shall calculate the integral limit in Proposition 5.2. Let $I_{m}(r)$ be as in (5.3). Recall from Corollary 4.9 that $u \in C^{\infty}(\mathbb{D})$. Consider the Taylor expansion of $u$ at the origin of degree $m \geq 0$ :

$$
u(z)=\sum_{\substack{j, k \geq 0 \\ j+k \leq m}} \frac{1}{j ! k !} \partial^{j} \bar{\partial}^{k} u(0) z^{j} \bar{z}^{k}+\mathcal{O}\left(|z|^{m+1}\right)
$$

as $z \rightarrow 0$. We have that

$$
\begin{aligned}
I_{m}(r) & =\frac{1}{2 \pi r^{m}} \int_{\mathbb{T}}\left(\sum_{\substack{j, k \geq 0 \\
j+k \leq m}} \frac{1}{j ! k !} \partial^{j} \bar{\partial}^{k} u(0) r^{j+k} e^{i(j-k) \theta}\right) e^{-i m \theta} d \theta+\mathcal{O}(r) \\
& =\partial^{m} u(0) / m !+\mathcal{O}(r)
\end{aligned}
$$

as $r \rightarrow 0$, where the last equality follows by cancellation. A passage to the limit as $r \rightarrow 0$ now yields that $c_{m}=\partial^{m} u(0) / m$ !.

We now turn to the formula for $c_{-m}$. A similar analysis as in the previous paragraph shows that

$$
I_{-m}(r)=\bar{\partial}^{m} u(0) / m !+\mathcal{O}(r)
$$

as $r \rightarrow 0$. A passage to the limit as $r \rightarrow 0$ yields that $c_{-m}=\bar{\partial}^{m} u(0) / m$ !. This completes the proof of the theorem.

The coefficient formulas in Theorem 5.3 leads to an addendum to Theorem 5.1.

Theorem 5.4 Let $p, q \in \mathbb{C}$. Let $u$ be $a(p, q)$-harmonic function. Then

$$
\begin{aligned}
u(z)= & \sum_{m=0}^{\infty} \frac{\partial^{m} u(0)}{m !} F\left(-p, m-q ; m+1 ;|z|^{2}\right) z^{m} \\
& +\sum_{m=1}^{\infty} \frac{\bar{\partial}^{m} u(0)}{m !} F\left(-q, m-p ; m+1 ;|z|^{2}\right) \bar{z}^{m}
\end{aligned}
$$

for $z \in \mathbb{D}$, where $F$ is the hypergeometric function (0.5).

Proof Recall the series expansion (5.1) and (5.2) established in Theorem 5.1. By Theorem 5.3 we have that $c_{m}=\partial^{m} u(0) / m$ ! and $c_{-m}=\bar{\partial}^{m} u(0) / m$ ! for $m \in \mathbb{N}$. This yields the conclusion of the theorem. 
As a by-product of Theorem 5.4 we have a uniqueness result of classical type.

Corollary 5.5 Let $p, q \in \mathbb{C}$. Let $u$ be $a(p, q)$-harmonic function. Assume that $u(0)=0$ and

$$
\partial^{m} u(0)=\bar{\partial}^{m} u(0)=0
$$

for $m=1,2, \ldots$ Then $u(z)=0$ for all $z \in \mathbb{D}$.

Proof The result is evident by Theorem 5.4.

\section{Further properties of the function $u_{p, q}$}

Let $u_{p, q}$ be as in (0.2) for some $p, q \in \mathbb{C}$. In this section we derive the series expansion of $u_{p, q}$ (see Theorem 6.3). Of interest are also properties of integral means of $u_{p, q}$ (see Theorems 6.4 and 6.6).

Lemma 6.1 Let $u_{p, q}$ be as in (0.2) for some $p, q \in \mathbb{C}$. Let $m \in \mathbb{N}$. Then

$$
\partial^{m} u_{p, q}(z)=\frac{(p+1)_{m}}{(1-z)^{m}} u_{p, q}(z)+\bar{z} g_{m}(z), \quad z \in \mathbb{D},
$$

where $g_{m} \in C^{\infty}(\mathbb{D})$.

Proof We prove the lemma by induction on $m \in \mathbb{N}$. Clearly (6.1) holds for $m=0$ with $g_{0}=0$. By Lemma 1.1 we have that

$$
\partial u_{p, q}(z)=\frac{p+1}{1-z} u_{p, q}(z)+\bar{z} g_{1}(z), \quad z \in \mathbb{D},
$$

with

$$
g_{1}(z)=-\frac{p+q+1}{1-|z|^{2}} u_{p, q}(z), \quad z \in \mathbb{D}
$$

This proves (6.1) for $m=1$.

Assume next that (6.1) holds for some $m=n \geq 1$, that is,

$$
\partial^{n} u_{p, q}(z)=\frac{(p+1)_{n}}{(1-z)^{n}} u_{p, q}(z)+\bar{z} g_{n}(z), \quad z \in \mathbb{D}
$$

where $g_{n} \in C^{\infty}(\mathbb{D})$. Differentiating we have that

$$
\partial^{n+1} u_{p, q}(z)=(p+1)_{n} \frac{n}{(1-z)^{n+1}} u_{p, q}(z)+\frac{(p+1)_{n}}{(1-z)^{n}} \partial u_{p, q}(z)+\bar{z} \partial g_{n}(z)
$$


for $z \in \mathbb{D}$. We next use (6.2) to see that

$$
\begin{aligned}
\partial^{n+1} u_{p, q}(z)= & (p+1)_{n} \frac{n}{(1-z)^{n+1}} u_{p, q}(z)+\frac{(p+1)_{n}}{(1-z)^{n}}\left(\frac{p+1}{1-z} u_{p, q}(z)+\bar{z} g_{1}(z)\right) \\
& +\bar{z} \partial g_{n}(z)=\frac{(p+1)_{n+1}}{(1-z)^{n+1}} u_{p, q}(z)+\bar{z} g_{n+1}(z)
\end{aligned}
$$

for $z \in \mathbb{D}$, where

$$
g_{n+1}(z)=\frac{(p+1)_{n}}{(1-z)^{n}} g_{1}(z)+\partial g_{n}(z), \quad z \in \mathbb{D} .
$$

This proves (6.1) for $m=n+1$. The conclusion of the lemma now follows by the principle of induction.

Notice that formula (6.1) interprets naturally as a congruence in $C^{\infty}(\mathbb{D})$ modulo $\bar{z} C^{\infty}(\mathbb{D})$.

Theorem 6.2 Let $u_{p, q}$ be as in (0.2) for some $p, q \in \mathbb{C}$. Then

$$
\partial^{m} u_{p, q}(0)=(p+1)_{m} \text { and } \bar{\partial}^{m} u_{p, q}(0)=(q+1)_{m}
$$

for $m \in \mathbb{N}$.

Proof We shall use Lemma 6.1. A point evaluation at the origin in (6.1) shows that $\partial^{m} u_{p, q}(0)=(p+1)_{m}$ for $m \in \mathbb{N}$. Moreover, by hermitian symmetry $\bar{u}_{p, q}=u_{\bar{q}, \bar{p}}$ we have that

$$
\bar{\partial}^{m} u_{p, q}(0)=\bar{\partial}^{m} \bar{u}_{\bar{q}, \bar{p}}(0)=\overline{\partial^{m} u_{\bar{q}, \bar{p}(0)}}=\overline{(\bar{q}+1)_{m}}=(q+1)_{m}
$$

for $m \in \mathbb{N}$.

We can now derive the series expansion of the function $u_{p, q}$.

Theorem 6.3 Let $p, q \in \mathbb{C}$. Then

$$
\begin{aligned}
\frac{\left(1-|z|^{2}\right)^{p+q+1}}{(1-z)^{p+1}(1-\bar{z})^{q+1}}= & \sum_{m=0}^{\infty} \frac{(p+1)_{m}}{m !} F\left(-p, m-q ; m+1 ;|z|^{2}\right) z^{m} \\
& +\sum_{m=1}^{\infty} \frac{(q+1)_{m}}{m !} F\left(-q, m-p ; m+1 ;|z|^{2}\right) \bar{z}^{m}
\end{aligned}
$$

for $z \in \mathbb{D}$, where $F$ is the hypergeometric function (0.5). 
Proof Let $u_{p, q}$ be as in (0.2). From Theorem 1.4 we know that the function $u_{p, q}$ is $(p, q)$-harmonic. By Theorem 5.4 it has the function series expansion

$$
\begin{aligned}
u_{p, q}(z)= & \sum_{m=0}^{\infty} \frac{\partial^{m} u_{p, q}(0)}{m !} F\left(-p, m-q ; m+1 ;|z|^{2}\right) z^{m} \\
& +\sum_{m=1}^{\infty} \frac{\bar{\partial}^{m} u_{p, q}(0)}{m !} F\left(-q, m-p ; m+1 ;|z|^{2}\right) \bar{z}^{m}
\end{aligned}
$$

for $z \in \mathbb{D}$. The result now follows from Theorem 6.2.

We point out that Theorem 6.3 generalizes a well-known partial fraction decomposition formula for the classical Poisson kernel for $\mathbb{D}$, see formula (1.1). Suitably specialized Theorem 6.3 yields [21, Theorem 2.5] and [18, Theorem 3.2].

Notice that the series expansion in Theorem 6.3 is absolutely convergent in $C^{\infty}(\mathbb{D})$.

We denote by $\operatorname{Re}(z)$ and $\operatorname{Im}(z)$ the real and imaginary parts of a complex number $z$, respectively. The standard Gamma function is defined by

$$
\Gamma(z)=\int_{0}^{\infty} t^{z-1} e^{-t} d t
$$

for $\operatorname{Re}(z)>0$. It is well-known that $\Gamma$ continues to a meromorphic function in $\mathbb{C}$ with simple poles at the points $z=0,-1,-2, \ldots$.

We next turn to $L^{1}$ means of $u_{p, q}$.

Theorem 6.4 Let $u_{p, q}$ be as in (0.2) for some $p, q \in \mathbb{C}$ such that $\operatorname{Re}(p)+\operatorname{Re}(q)>-1$. Then

$$
\frac{1}{2 \pi} \int_{\mathbb{T}}\left|u_{p, q}\left(r e^{i \theta}\right)\right| d \theta \leq e^{\frac{\pi}{2}|\operatorname{Im}(p)-\operatorname{Im}(q)|} \frac{\Gamma(\operatorname{Re}(p)+\operatorname{Re}(q)+1)}{\Gamma\left(\frac{\operatorname{Re}(p)+\operatorname{Re}(q)}{2}+1\right)^{2}}
$$

for $0 \leq r<1$, where $\Gamma$ is the Gamma function.

Proof From definition of powers we have that

$$
u_{p, q}(z)=e^{(p+q+1) \log \left(1-|z|^{2}\right)} e^{-(p+q+2) \log |1-z|} e^{-i(p-q) \arg (1-z)}
$$

for $z \in \mathbb{D}$. Notice that the complex number $1-z$ lies in the open right half-plane when $z \in \mathbb{D}$. Passing to absolute values we have that

$$
\left|u_{p, q}(z)\right| \leq e^{\frac{\pi}{2}|\operatorname{Im}(p)-\operatorname{Im}(q)|} \frac{\left(1-|z|^{2}\right)^{\operatorname{Re}(p)+\operatorname{Re}(q)+1}}{|1-z|^{\operatorname{Re}(p)+\operatorname{Re}(q)+2}}
$$

for $z \in \mathbb{D}$. We next apply [18, Theorem 3.1] with $\alpha=\operatorname{Re}(p)+\operatorname{Re}(q)$ to conclude that

$$
\frac{1}{2 \pi} \int_{\mathbb{T}}\left|u_{p, q}\left(r e^{i \theta}\right)\right| d \theta \leq e^{\frac{\pi}{2}|\operatorname{Im}(p)-\operatorname{Im}(q)|} \frac{\Gamma(\alpha+1)}{\Gamma(\alpha / 2+1)^{2}}
$$


for $0 \leq r<1$. This yields the conclusion of the theorem.

Remark 6.5 A similar analysis as in the proof of Theorem 6.4 shows that

$$
\lim _{r \rightarrow 1} \frac{1}{2 \pi} \int_{\mathbb{T}}\left|u_{p, q}\left(r e^{i \theta}\right)\right| d \theta=+\infty
$$

if $\operatorname{Re}(p)+\operatorname{Re}(q) \leq-1$. We omit the details.

A classical result known as Gauss summation formula says that

$$
\lim _{x \rightarrow 1} F(a, b ; c ; x)=\frac{\Gamma(c) \Gamma(c-a-b)}{\Gamma(c-a) \Gamma(c-b)}
$$

when $a, b \in \mathbb{C}$ and $c \in \mathbb{C} \backslash\{0,-1,-2, \ldots\}$ are such that $\operatorname{Re}(c)>\operatorname{Re}(a)+\operatorname{Re}(b)$ (see [3, Theorem 2.2.2]).

Theorem 6.6 Let $p, q \in \mathbb{C}$ be such that $\operatorname{Re}(p)+\operatorname{Re}(q)>-1$. Then

$$
\lim _{r \rightarrow 1} \frac{1}{2 \pi} \int_{\mathbb{T}} u_{p, q}\left(r e^{i \theta}\right) d \theta=\frac{\Gamma(p+q+1)}{\Gamma(p+1) \Gamma(q+1)},
$$

where $\Gamma$ is the Gamma function.

Proof Recall the series expansion of the function $u_{p, q}$ established in Theorem 6.3. By cancellation of terms we have that

$$
\frac{1}{2 \pi} \int_{\mathbb{T}} u_{p, q}\left(r e^{i \theta}\right) d \theta=F\left(-p,-q ; 1 ; r^{2}\right)
$$

for $0 \leq r<1$. Since $\operatorname{Re}(p)+\operatorname{Re}(q)>-1$, we can apply Gauss summation formula (6.3) to calculate the limit of the above quantity as $r \rightarrow 1$. This yields the conclusion of the theorem.

\section{The Dirichlet problem: concluding remarks}

In a restricted range of parameters, the class of $(p, q)$-harmonic functions can be analyzed in terms of their boundary values. We shall discuss in this section some rudiments of such theory.

Let $p, q \in \mathbb{C} \backslash \mathbb{Z}^{-}$be such that $\operatorname{Re}(p)+\operatorname{Re}(q)>-1$. The $(p, q)$-harmonic Poisson kernel is defined by

$$
K_{p, q}(z)=c_{p, q} \frac{\left(1-|z|^{2}\right)^{p+q+1}}{(1-z)^{p+1}(1-\bar{z})^{q+1}}, \quad z \in \mathbb{D},
$$

where

$$
c_{p, q}=\frac{\Gamma(p+1) \Gamma(q+1)}{\Gamma(p+q+1)} .
$$


Notice that the constant $c_{p, q}$ is a non-zero complex number in this range of parameters. By Theorem 1.4 the function $K_{p, q}$ is $(p, q)$-harmonic. Theorems 6.4 and 6.6 ensure that the function $K_{p, q}$ satisfies some standard properties for approximate identities.

The $(p, q)$-harmonic Poisson integral is defined by

$$
K_{p, q}[f](z)=\frac{1}{2 \pi} \int_{\mathbb{T}} K_{p, q}\left(z e^{-i \theta}\right) f\left(e^{i \theta}\right) d \theta, \quad z \in \mathbb{D},
$$

for integrable functions $f \in L^{1}(\mathbb{T})$ on $\mathbb{T}$.

Let $C(\mathbb{T})$ be the space of continuous functions on $\mathbb{T}$ and fix $\varphi \in C(\mathbb{T})$. By the $(p, q)$-harmonic Dirichlet problem for $\varphi$ we understand the problem of finding a $(p, q)$-harmonic function $u$ such that $\lim _{r \rightarrow 1} u_{r}=\varphi$ in $C(\mathbb{T})$, where

$$
u_{r}\left(e^{i \theta}\right)=u\left(r e^{i \theta}\right), \quad e^{i \theta} \in \mathbb{T},
$$

for $0<r<1$. Following usual practice, we formulate this latter Dirichlet problem as

$$
\left\{\begin{array}{c}
L_{p, q} u=0 \text { in } \mathbb{D} \\
u=\varphi \text { on } \mathbb{T}
\end{array}\right.
$$

where $L_{p, q}$ is as in (0.1).

Theorem 7.1 Let $p, q \in \mathbb{C} \backslash \mathbb{Z}^{-}$be such that $\operatorname{Re}(p)+\operatorname{Re}(q)>-1$. Let $\varphi \in C(\mathbb{T})$. Then a function $u$ in $\mathbb{D}$ satisfies (7.1) if and only if it has the form

$$
u(z)=K_{p, q}[\varphi](z), \quad z \in \mathbb{D} .
$$

The proof of Theorem 7.1 follows a standard scheme for such results and is therefore omitted, see $[18,21]$ for details.

The present paper suggests a finer study of $(p, q)$-harmonic functions. Earlier results of such type concern Poisson integral representations, pointwise boundary limits, Green functions and Lipschitz continuity of generalized harmonic functions, see [18-21]. We mention here also work of Ahern and collaborators [1,2].

Funding Open access funding provided by Lund University.

\section{Declarations}

Conflict of interest The authors declare that they have no conflict of interest.

Open Access This article is licensed under a Creative Commons Attribution 4.0 International License, which permits use, sharing, adaptation, distribution and reproduction in any medium or format, as long as you give appropriate credit to the original author(s) and the source, provide a link to the Creative Commons licence, and indicate if changes were made. The images or other third party material in this article are included in the article's Creative Commons licence, unless indicated otherwise in a credit line to the material. If material is not included in the article's Creative Commons licence and your intended use is not permitted 
by statutory regulation or exceeds the permitted use, you will need to obtain permission directly from the copyright holder. To view a copy of this licence, visit http://creativecommons.org/licenses/by/4.0/.

\section{References}

1. Ahern, P., Bruna, J., Cascante, C.: $H^{p}$-theory for generalized $\mathcal{M}$-harmonic functions in the unit ball. Indiana Univ. Math. J. 45, 103-135 (1996)

2. Ahern, P., Cascante, C.: Exceptional sets for Poisson integrals of potentials on the unit sphere in $\mathbb{C}^{n}$, $p \leq 1$. Pac. J. Math. 153, 1-13 (1992)

3. Andrews, G.E., Askey, R., Roy, R.: Special Functions. Encyclopedia of Mathematics and its Applications, vol. 71. Cambridge University Press, Cambridge (1999)

4. Astala, K., Päivärinta, L.: Calderón's inverse conductivity problem in the plane. Ann. Math. 163, 265-299 (2006)

5. Behm, G.: Solving Poisson's equation for the standard weighted Laplacian in the unit disc (2014). arXiv:1306.2199v2 [math.AP]

6. Borichev, A., Hedenmalm, H.: Weighted integrability of polyharmonic functions. Adv. Math. 264, 464-505 (2014)

7. Calderón, A.P.: On an inverse boundary value problem, Seminar on Numerical Analysis and its Applications to Continuum Physics (Rio de Janeiro, 1980), pp. 65-73, Soc. Brasil. Mat., Rio de Janeiro, : reprinted in Comput. Appl. Math. 25(2006), 133-138 (1980)

8. Carlsson, M., Wittsten, J.: The Dirichlet problem for standard weighted Laplacians in the upper half plane. J. Math. Anal. Appl. 436, 868-889 (2016)

9. Chen, S., Vuorinen, M.: Some properties of a class of elliptic partial differential operators. J. Math. Anal. Appl. 431, 1124-1137 (2015)

10. Chen, X., Kalaj, D.: A representation theorem for standard weighted harmonic mappings with an integer exponent and its applications. J. Math. Anal. Appl. 444, 1233-1241 (2016)

11. Conway, J.B.: Functions of One Complex Variable, second edition, Graduate Texts in Mathematics, vol. 11. Springer, New York (1978)

12. Garabedian, P.R.: A partial differential equation arising in conformal mapping. Pac. J. Math. 1, 485-524 (1951)

13. Geller, D.: Some results in $H^{p}$ theory for the Heisenberg group. Duke Math. J. 47, 365-390 (1980)

14. Hedenmalm, H.: On the uniqueness theorem of Holmgren. Math. Z. 281, 357-378 (2015)

15. Katznelson, Y.: An Introduction to Harmonic Analysis. Dover, New York City (1976)

16. Li, P., Wang, X.: Lipschitz continuity of $\alpha$-harmonic functions. Hokkaido Math. J. 48, 85-97 (2019)

17. Li, P., Wang, X., Xiao, Q.: Several properties of $\alpha$-harmonic functions in the unit disk. Monatsh. Math. 184, 627-640 (2017)

18. Olofsson, A.: Differential operators for a scale of Poisson type kernels in the unit disc. J. Anal. Math. 123, 227-249 (2014)

19. Olofsson, A.: On a weighted harmonic Green function and a theorem of Littlewood. Bull. Sci. Math. 158, 102809 (2020)

20. Olofsson, A.: Lipschitz continuity for weighted harmonic functions in the unit disc. Complex Var. Elliptic Equ. 65, 1630-1660 (2020)

21. Olofsson, A., Wittsten, J.: Poisson integrals for standard weighted Laplacians in the unit disc. J. Math. Soc. Jpn. 65, 447-486 (2013)

22. Wittsten, J.: Generalized axially symmetric potentials with distributional boundary values. Bull. Sci. Math. 139, 892-922 (2015)

Publisher's Note Springer Nature remains neutral with regard to jurisdictional claims in published maps and institutional affiliations. 